\title{
Ligand exchange method for determination of mole ratios of relatively weak metal complexes: a comparative study
}

\author{
Mokhtar Mabrouk ${ }^{1,2}$, Sherin F. Hammad', Mohamed A. Abdelaziz ${ }^{1,3}$ and Fotouh R. Mansour ${ }^{1,2^{*}}$
}

\begin{abstract}
Ligand exchange method is introduced as an alternative to Job's and mole ratio methods for studying the stoichiometry of relatively weak metal complexes in solutions. The method involves adding varying amounts of a ligand $(L)$ to an excess constant amount of a colored complex (MX) with appropriate stability and molar absorptivity. The absorbance of each solution is measured at the $\lambda_{\max }$ of the initial complex, $M X$, and plotted against the concentration of the studied ligand, L. If the newly formed complex ML does not absorb at the $\lambda_{\max }$ of the initial complex, then attenuation of the absorbance of the initial complex on adding varying quantities of the investigational ligand gives an inverse calibration line that intersects with the calibration curve of initial complex at a given point. If a line parallel to the ordinate is drawn from this point to the $x$-axis, the ratio of the two parts of the $x$-axis to the left and to the right $(\alpha / \beta)$ gives the metal to ligand molar ratio in the complex formed, ML. The new method has been applied to the study of the composition of iron (III) complexes with three bisphosphonate drugs: alendronate, etidronate, and ibandronate. The mole ratio was found to be 1:1 with the three investigated bisphosphonates and results were further confirmed by Job's and mole ratio methods. The ligand exchange method is simpler, quicker, easier to perform and more accurate than Job's and mole ratio methods for studying weak and relatively weak complexes.
\end{abstract}

Keywords: Ligand exchange method, Mole ratio method, Job's method, Bisphosphonates, Relatively weak complexes

\section{Introduction}

The mole ratio is the proportion of number of moles of any two chemical entities involved in a compound or a chemical reaction. Studying the mole ratio is important to calculate the reaction yield, determine the stoichiometry and monitor the reaction kinetics. Several spectrophotometric methods were developed for the determination of the molar ratio of metal complexes. The first method goes back to the contributions of Ostromisslensky [1] and Job [2], and was widely known as Job's method of continuous variations. In this method, a series of solutions are prepared by mixing varying proportions of the metal and ligand, keeping the sum of the total molar concentrations

\footnotetext{
*Correspondence: fotouhrashed@pharm.tanta.edu.eg

2 Pharmaceutical Services Center, Faculty of Pharmacy, Tanta University, Tanta 31111, Egypt

Full list of author information is available at the end of the article
}

constant. The absorbance of each solution is then plotted against the mole fraction of either the ligand or metal. The position of the maximum in the resulting curve, or minimum in some cases [3], gives the mole fraction. The simplicity of the method made it widely applied for the study of various metals and association complexes [4-9], in spite of its limitations. For instance, strong complexes give triangular plots from which the position of the maximum is easily determined, while the plots of weak complexes are highly curved leading to unreliable results. Normalized absorbance plots ( $\mathrm{A} / \mathrm{A}_{\max }$ vs. mole fraction) gave sharper plots at the maxima and allowed for better location of the mole ratio [10], but for weak complexes, these normalized Job plots were still highly curved.

Besides the method of continuous variations, the mole ratio method has been used frequently since its introduction by Yoe and Jones [11]. In this method, a series of solutions are prepared by varying the amount of ligand 
in each solution while the amount of metal is kept constant. If a stable complex is formed, a plot of absorbance versus mole ratio of ligand to metal $(\mathrm{L} / \mathrm{M})$ gives a straight line that rises until it reaches the point corresponding to the mole ratio $(\mathrm{L} / \mathrm{M})$, then it breaks to a differently sloped line. For moderately stable complex, the mole ratio corresponds to the point of intersection of the tangents of straight-line portions of the plot. However, if a weak complex is formed, a very curved plot is obtained, making the identification of the molar ratio of these complexes uncertain. As a result, several chemical [12] and mathematical modifications [13-15] have been made to the basic mole ratio method so that it can reliably be applied to study the composition of weak complexes. However, these modifications make the method relatively more complicated and are only applicable when the ligand has significant absorbance which is not always the case.

A recent method based on ligand exchange has been introduced by Mansour and Danielson [16]. The method involves adding varying amounts of the ligand $(\mathrm{L})$, whose combining ratio with metal $(\mathrm{M})$ is being studied, to an excess constant amount of a colored complex (MX) with appropriate stability and molar absorptivity. The absorbance of each solution is measured at the $\lambda_{\max }$ of the initial complex, MX, and plotted against the concentration of the studied ligand, L. If the newly formed complex, ML, does not absorb at the $\lambda_{\max }$ of the initial complex, then attenuation of the absorbance of the initial complex on adding varying quantities of the investigational ligand gives an inverse calibration line that intersects with the calibration curve of initial complex at a given point (Fig. 1). If a line parallel to the ordinate is drawn from this point to the $\mathrm{x}$-axis, the ratio of the two parts of the $x$-axis to the left and to the right $(\alpha / \beta)$ gives the metal to ligand molar ratio in the complex formed. A video that explains the principle of Mansour-Danielson's method is shown in Additional file 1.

In our previous work, the ligand exchange method has been applied for determination of mole ratios other than 1:1 [16]. In this work, we present the mathematical proof of the ligand exchange method for the first time and apply it for determination of relatively weak complexes of selected bisphosphantes (Fig. 2) with ferric ion [9]. The ferric complexes of bisphosphonates are used for the spectrophotometric determination of bisphosphonates in pharmaceutical tablets [9]. Determination of the mole ratios of these complexes is important to adjust the amount added of the ferric salt in the experimental part. The ligand exchange method was also compared with Job's and mole ratio methods; its advantages over these commonly employed methods are discussed.

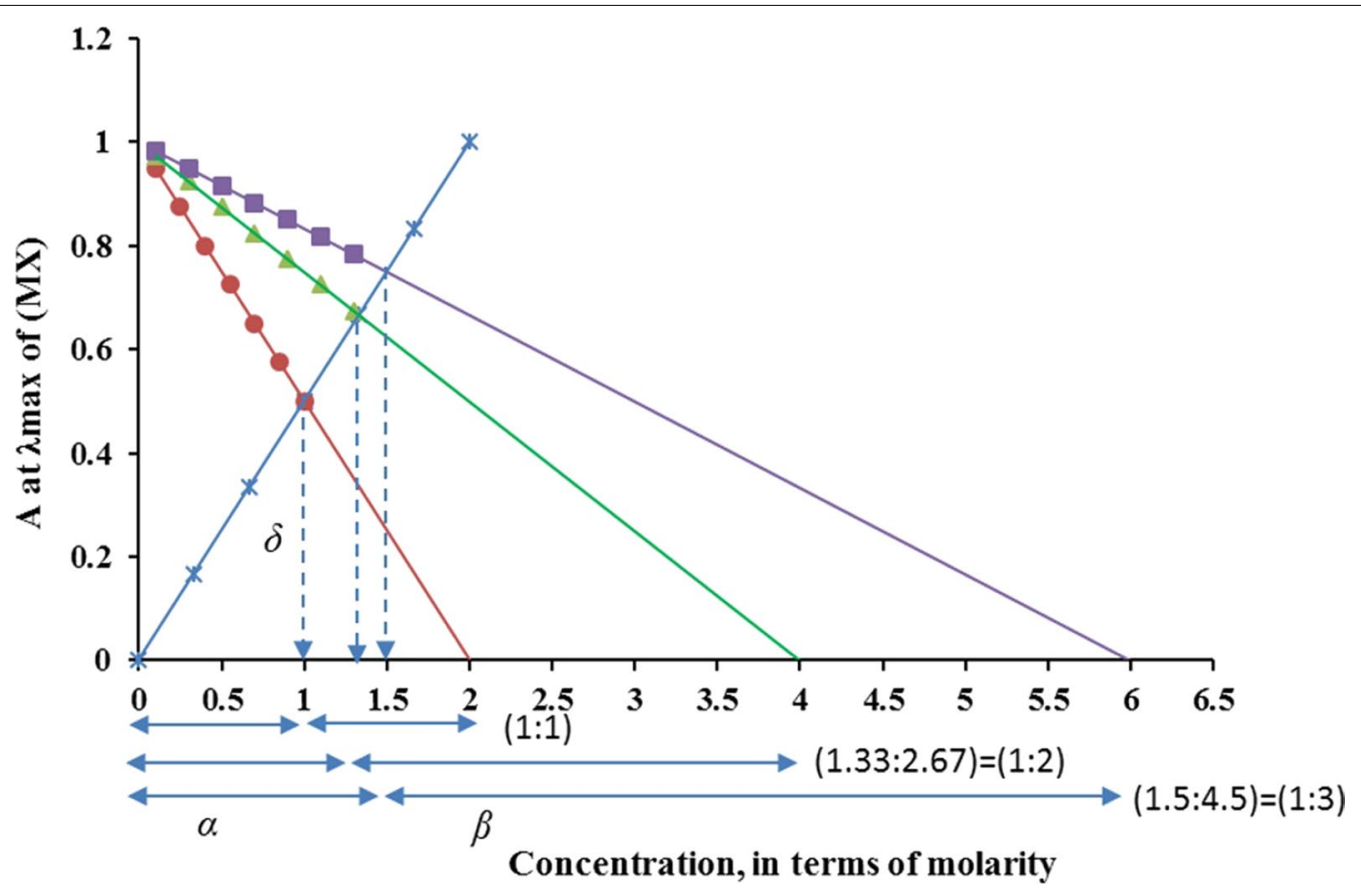

Fig. 1 Illustrative plots of the ligand exchange method using $M X$ as an initial complex $\left.{ }^{*}\right)$ for studying the mole ratios of complexes: $M L(\mathbf{O}), M L_{2}$ (), and $\mathrm{ML}_{3}$ 
<smiles>CCCCCN(C)CCC(O)(P(=O)(O)O)P(=O)(O)O</smiles>

Etidronate Disodium

Alendronate Sodium

Ibandronate Sodium

Fig. 2 Molecular structures of studied bisphosphonate drugs. All compounds are presented in anhydrous forms

\section{Theory of Mansour-Danielson's method of ligand exchange}

Suppose that MX and ML are two complexes of a metal $\mathrm{M}$ with two ligands, $\mathrm{X}$ and $\mathrm{L}$, where $\mathrm{MX}$ is a colored complex, ML is a colorless complex and MX is less stable than ML. For a certain concentration of the complex MX, the absorbance depends on the molar absorptivity of MX $\left(\varepsilon_{M X}\right)$ and the concentration $\left(C_{M X}\right)$ according to the equation:

$$
A=\varepsilon_{M X} \cdot C_{M X}
$$

If a certain amount of ligand $\mathrm{L}$ was added to the previous MX solution, a displacement reaction will take place and the absorbance will decrease as shown in Fig. 1. The decrease in the absorbance depends on the concentration of the ligand $\mathrm{L}\left(C_{L}\right)$ and the mole ratio of the ML complex $(n)$ according to the equation:

$$
A=\varepsilon_{M X} \cdot\left(C_{M X}-n C_{L}\right)
$$

From Eq. 2, we get:

$$
A=\varepsilon_{M X} \cdot C_{M X}-n \varepsilon_{M X} \cdot C_{L}
$$

Equation 3 is a straight line equation $(\mathrm{y}=\mathrm{a} \pm \mathrm{bx})$ with an intercept equals $\varepsilon_{M X} \cdot C_{M X}$ and a slope equals $-n \cdot \varepsilon_{M X}$. If $A$ was plotted against $C_{L}$, a straight line with a negative slope will be obtained as shown in Fig. 1. The mole ratio can be determined graphically from the overlay of the two calibration curves as follows:

A straight line parallel to the $y$-axis is drawn from the intersection point of the calibration curves to divide the $\mathrm{x}$-axis into two parts: $\alpha$ and $\beta$. The length of both parts $(\alpha$ and $\beta$ ) can be calculated from the length of the parallel line $(\delta)$ and the slopes of the calibration curves where:

$$
\alpha=\frac{\delta}{\text { Slope of } E q 1}=\frac{\delta}{\varepsilon_{\mathrm{MX}}}
$$

while,

$$
\beta=\frac{\delta}{\text { Slope of } E q 2}=\frac{\delta}{n \varepsilon_{\mathrm{MX}}}
$$

From Eqs. 4 and 5, we get:

$$
\frac{\alpha}{\beta}=n
$$

\section{Experimental \\ Instrumentation}

Jenway 3510 (Jenway, UK) and Biochrom libra S80 (Biochrom, Cambridge, UK) were employed in all $\mathrm{pH}$ and absorbance measurements, respectively.

\section{Materials}

Alendronate sodium trihydrate, etidronate disodium, and ibandronate sodium monohydrate of pharmaceutical grade were kindly provided by Sigma Pharmaceutical Industries (Quesna, Menofyia, Egypt). All other chemicals and solvents used were of analytical ACS grade, purchased from Fisher Scientific (Fair Lawn, NJ, USA) and Sigma-Aldrich (St. Louis, MO, USA).

\section{Standard solutions}

$\mathrm{Fe}(\mathrm{III})$-salicylate solution was prepared at $10 \mathrm{mM}$ in water/methanol (50:50, $\mathrm{pH} 3.2$ ) and was proved to be stable for months when kept refrigerated. Fe(III) chloride stock solution (for the mole ratio and Job's methods) was prepared at $10 \mathrm{mM}$ in $2 \mathrm{M} \mathrm{HClO}_{4}$. Etidronate disodium stock solution was prepared at $10 \mathrm{mM}$ in two different diluents: $2 \mathrm{M} \mathrm{HClO}_{4}$ for both the mole ratio and Job's methods and water/methanol (50:50, $\mathrm{pH} 3.2)$ for the ligand exchange method. Similarly, stock solutions of alendronate sodium and ibandronate sodium were prepared.

\section{Procedures}

\section{Ferric salicylate complex calibration curve}

A series of standard solutions of ferric salicylate in the range of $0.1-0.6 \mathrm{mM}$ were prepared by accurately transferring appropriate aliquots of ferric salicylate stock solution $(10 \mathrm{mM})$ into a series of $10 \mathrm{~mL}$ calibrated volumetric flasks, then completed to the mark with water/methanol 
(50:50, pH 3.2) (Ionic strength was adjusted with $0.5 \mathrm{M}$ $\mathrm{NaCl})$. Absorbance at $535 \mathrm{~nm}$ was measured and plotted against ferric salicylate concentration.

\section{Ligand exchange method}

Aliquots in the range $0.2-1.8 \mu \mathrm{mol}$ of etidronate disodium were accurately transferred into a series of $10 \mathrm{~mL}$ volumetric flasks containing $3 \mu \mathrm{mol}$ ferric salicylate, then completed to the mark with water/methanol (50:50, pH 3.2) (Ionic strength was adjusted with $0.5 \mathrm{M}$ $\mathrm{NaCl})$. Absorbance at $535 \mathrm{~nm}$ was measured and plotted against concentration. A similar procedure was applied to determine the mole ratio of $\mathrm{Fe}(\mathrm{III})$-alendronate and Fe(III)-ibandronate.

\section{Job's method}

Standard nine mixtures of ferric chloride (in $2 \mathrm{M} \mathrm{HClO}_{4}$ ) and etidronate (in $2 \mathrm{M} \mathrm{HClO}_{4}$ ) were prepared by adding aliquots of $\mathrm{Fe}(\mathrm{III})$ equivalent to $1-9 \mu \mathrm{mol}$ into a series of $10 \mathrm{~mL}$ volumetric flasks containing aliquots of etidronate equivalent to 9-1 $\mu \mathrm{mol}$ so that each flask contains a total number of $10 \mu \mathrm{mol}$. Each flask is completed to the mark using $\mathrm{HClO}_{4}(2 \mathrm{M})$. Job's graph is obtained by plotting absorbance at $300 \mathrm{~nm}$ against the mole fraction of Fe(III) ion. The same procedure was repeated with ibandronate and alendronate.

\section{Mole ratio method}

Standard mixtures of ferric chloride (in $2 \mathrm{M} \mathrm{HClO}_{4}$ ) and etidronate (in $2 \mathrm{M} \mathrm{HClO}_{4}$ ) were prepared by adding aliquots of Fe(III) equivalent to $0.4-30 \mu \mathrm{mol}$ into a series of $10 \mathrm{~mL}$ volumetric flasks containing $5 \mu \mathrm{mol}$ of etidronate. Each flask is completed to the mark using $\mathrm{HClO}_{4}(2 \mathrm{M})$. The mole ratio graph is obtained by plotting absorbance at $300 \mathrm{~nm}$ against the mole ratio (Fe(III)/etidronate). The same procedure was applied to study the stoichiometry of Fe(III)-ibandronate and Fe(III)-alendronate.

\section{Results and discussion}

\section{Absorption spectra}

The absorption spectra of reacting species, Fe(III) ions and etidronate, together with the absorption spectrum of their complex have been recorded in $2 \mathrm{M}$ perchloric acid in the wavelength range from 200 to $400 \mathrm{~nm}$ (Fig. 3). Spectra of iron(III) perchlorate and iron(III)-etidronate complex show an absorption maximum at 239 and $252 \mathrm{~nm}$, respectively. On the other hand, etidronate and the other studied bisphosphonates do not show significant absorbance in the spectral region indicated above [17]. For Job's and mole ratio methods, all absorbance measurements were performed at $300 \mathrm{~nm}$ where the absorbance difference between the complex and Fe(III) ions approaches maximum, and the absorption of metal

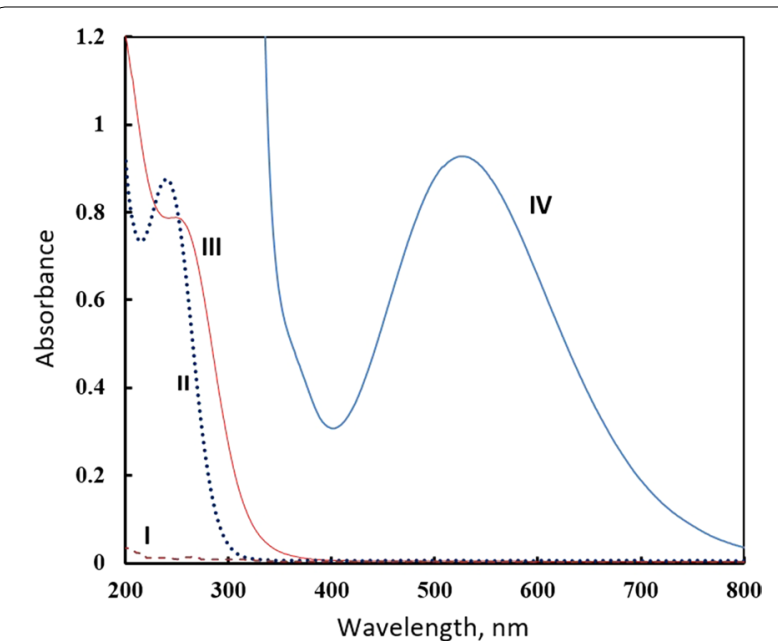

Fig. 3 Absorption spectra of (I) etidronate $\left(1 \times 10^{-3} \mathrm{M}\right)$, (II) $\mathrm{FeCl}_{3}$ $\left(2 \times 10^{-4} \mathrm{M}\right)$, and $(\mathrm{III}) \mathrm{FeCl}_{3}\left(2 \times 10^{-4} \mathrm{M}\right)+$ etidronate $\left(4 \times 10^{-4} \mathrm{M}\right)$ all in $2 \mathrm{M}$ perchloric in addition to (IV) the absorption Spectrum of Fe(III)-salicylate in water/methanol (50:50, pH 3.2)

ions is low. For the ligand exchange method, all spectrophotometric measurements were conducted at $535 \mathrm{~nm}$, the wavelength that corresponds to the absorption maximum of iron(III)-salicylate at the conditions employed.

\section{Ligand exchange method using Fe(III)-salicylate}

According to a previously published work that studied the effect of $\mathrm{pH}$ and ionic strength on the absorbance of Fe(III)-salicylate complex [18], the absorbance of the complex was found constant over a $\mathrm{pH}$ range of (2.5-3.5). After trying several solvents, a 50\% methanol at $\mathrm{pH} 3.2$ was chosen owing to the high Fe(III)-salicylate absorbance and reasonable plateau that ensures the robustness of the method against small changes in $\mathrm{pH}$. A solution of $0.5 \mathrm{M} \mathrm{NaCl}$ was used to adjust the ionic strength and keep it constant over all the following procedures.

An overlay of the direct and inverse calibration curves of ferric salicylate and bisphosphonate, respectively, is used to determine the combining metal to ligand ratio (Fig. 4). The quotient of $\alpha / \beta$ is equal to the stoichiometric ratio of metal to bisphosphonate ligand and was found to be 1:1 with the three investigated bisphosphonates. Calibration curves of the three studied bisphosphonates were linear in the range $(0.02-0.18) \mathrm{mM}$ with correlation coefficients $(r)$ equal $-0.999,-0.997$ and -0.996 with etidronate, alendronate, and ibandronate, respectively.

\section{Comparison to other mole ratio methods}

The 1:1 ratio determined for the Fe(III) complex with alendronate is congruent with the work of Kuljanin and his colleagues [9] that is based on Job's and mole ratio 

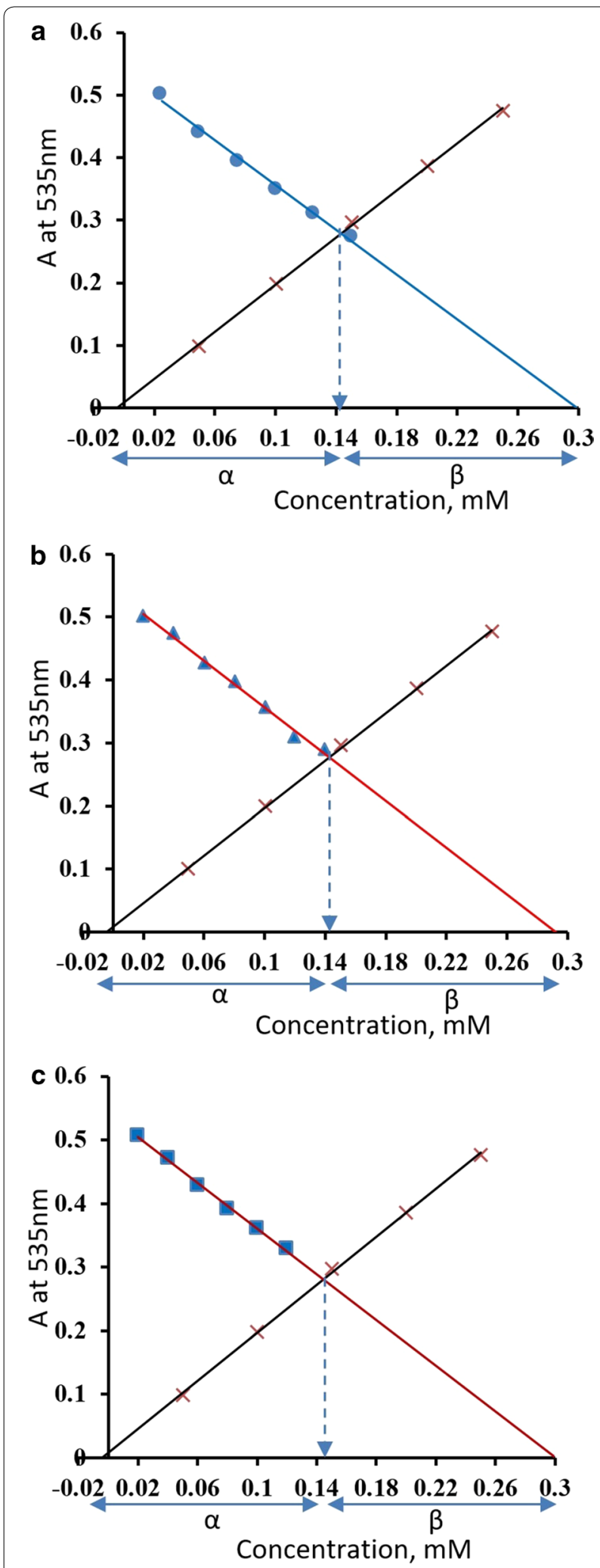

Fig. 4 An overlay of Fe(III)-salicylate calibration curve $(x)$ with inverse calibration curves of a ibandronate $(\mathbf{O})$, b alendronate $(\boldsymbol{\Lambda})$, and $\mathbf{c}$ etidronate
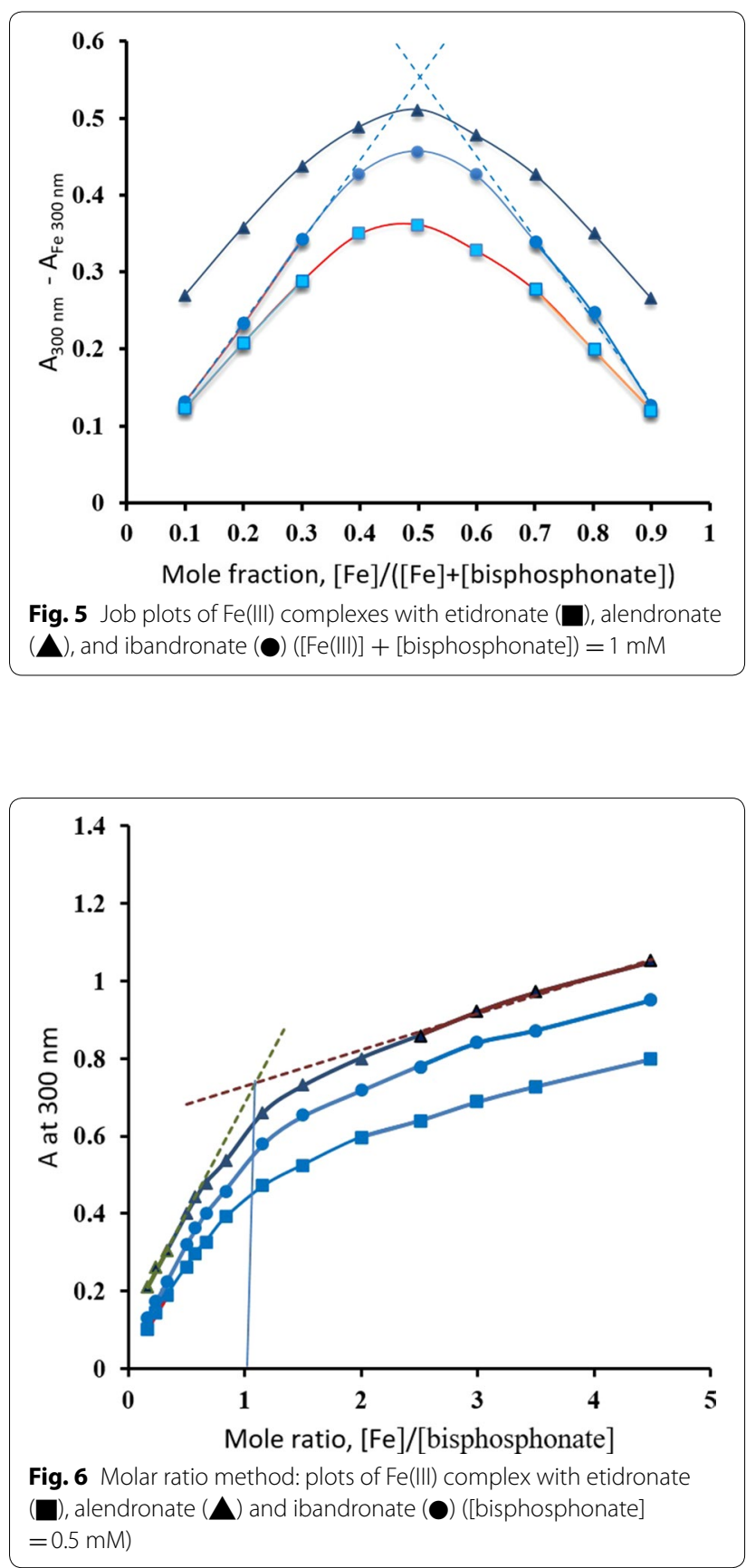

methods. On the other hand, results of ibandronate and etidronate complexes with $\mathrm{Fe}(\mathrm{III})$ have been confirmed by performing Job's and mole ratio methods. The Job's plots (Fig. 5) showed a peak at a mole fraction of 0.5, whereas the tangents of straight-line portions of the mole ratio curves intersect at a value of 1 (Fig. 6). Therefore, results of both methods provide a further confirmation of the 1:1 ratio determined by the ligand exchange method. 
Compared to the Job and mole ratio methods, the ligand exchange method offers several advantages: (i) it enables the study of the composition of colorless metal complexes using a colorimetric technique and the green LED lamp that is commercially available in most colorimeters (ii) it requires fewer steps than Job's and the mole ratio methods because fewer number of points can be adequate to plot a straight line and several ligands can be studied against a single calibration curve of the initial complex, (iii) the ligand exchange method is more accurate and more precise than Job's and the mole ratio methods for determination of weak and relatively weak complexes; determining the mole ratio using these methods in this case is subjective due to the curved lines. As shown in Additional file 2: Fig. S1, different tangents can be drawn for the same group of points, which may lead to false conclusions while in the ligand exchange method, there is no need to draw tangents which obviates bias and decreases the risk of error. (iv) The ligand exchange method could be used for metals other than ferric, such as $\mathrm{Cu}(\mathrm{II})$, and for determination of mole ratios other than 1:1 [16] which indicates the generality of the method and (v) neither Job's nor the mole ratio methods can be used unless one of the studied reactants or the formed complex are absorbing. In this case, the ligand exchange will be the method of choice.

\section{Conclusion}

The ligand exchange method can reliably be used as an alternative to Job's and mole ratio methods for the determination of formula of complexes with the aid of a simple colorimeter, and could be superior in determining the composition of weak and relatively weak complexes. The method has successfully been applied to the study of the composition of ferric ion complexes with the nonchromophoric bisphosphonates: alendronate, etidronate and ibandronate. The ligand exchange method gives straight lines from which the exact mole ratio can be determined. The method does not require tangent drawing which can be subjective and may lead to inaccurate conclusions especially when weak complexes are studied. The ligand exchange method could also be preferable for determining the composition of high ratio complexes and that will be the focus of our future research.

\section{Additional files}

Additional file 1: A video that explains the principle of MansourDanielson's method.Additional file 2: Fig. S1. Molar ratio's plots for Fe(III) complex with ibandronate showing different conclusions for the same results depending on the drawn tangents.
Additional file 2: Fig. S1. Molar ratio's plots for Fe(III) complex with ibandronate showing different conclusions for the same results depending on the drawn tangents.

\section{Authors' contributions}

MM participated in the study design and the results discussion and revised the manuscript. SFH participated in the study design and the results discussion and revised the manuscript. MAA conducted the practical work, participated in the results discussion and the preparation and writing of the manuscript. FRM proposed the study design, participated in the results discussion, literature review, manuscript preparation and revision. All authors read and approved the final manuscript.

\section{Author details}

${ }^{1}$ Department of Pharmaceutical Analytical Chemistry, Faculty of Pharmacy, Tanta University, Tanta 31111, Egypt. ${ }^{2}$ Pharmaceutical Services Center, Faculty of Pharmacy, Tanta University, Tanta 31111, Egypt. ${ }^{3}$ Department of Pharmaceutical Analytical Chemistry, Faculty of Pharmacy, Kafrelsheikh University, Kafrelsheikh 33511, Egypt.

\section{Competing interests}

The author declares that they have no competing interests.

Availability of data and materials

All data and materials are all provided.

Consent for publication

All the authors gave their consent for the publication of this article.

\section{Ethics approval and consent to participate}

The experiment was conducted according to the rules of the Ethical committee of the Tanta University, Egypt.

\section{Publisher's Note}

Springer Nature remains neutral with regard to jurisdictional claims in published maps and institutional affiliations.

Received: 9 May 2018 Accepted: 4 December 2018

Published online: 20 December 2018

\section{References}

1. Ostromisslensky I (1911) Über eine neue, auf dem Massenwirkungsgesetz fußende Analysenmethode einiger binärer Verbindungen. Zur Prioritätsfrage. Berichte der Dtsch Chem Gesellschaft 44:1189-1190. https://doi. org/10.1002/cber.191104401176

2. Job P (1928) Formation and stability of inorganic complexes in solution. Ann Chim 9:113

3. Vosburgh WC, Cooper GR (1941) Complex lons. I. The identification of complex ions in solution by spectrophotometric measurements. J Am Chem Soc 63:437-442. https://doi.org/10.1021/ja01847a025

4. Qin Z, Niu W, Tan R (2009) Spectrophotometric method for the determination of telmisartan with congo red. J Anal Chem 64:449-454. https:// doi.org/10.1134/S1061934809050049

5. Zayed SIM (2009) Two charge-transfer complex spectrophotometric methods for the determination of sulpiride in pharmaceutical formulations. Cent Eur J Chem 7:870-875. https://doi.org/10.2478/s1153 2-009-0091-2

6. Shaalan RA-A (2010) Improved spectrofluorimetric methods for determination of penicillamine in capsules. Cent Eur J Chem 8:892-898. https:// doi.org/10.2478/s11532-010-0049-4

7. Nagaraj P, Aradhana N, Shivakumar A et al (2009) Spectrophotometric method for the determination of chromium (VI) in water samples. Environ Monit Assess 157:575-582. https://doi.org/10.1007/s1066 $1-008-0557-2$ 
8. Ostović D, Stelmach C, Hulshizer B (1993) Formation of a chromophoric complex between alendronate and copper(II) ions. Pharm Res 10:470-472

9. Kuljanin J, Janković I, Nedeljković J et al (2002) Spectrophotometric determination of alendronate in pharmaceutical formulations via complex formation with Fe(III) ions. J Pharm Biomed Anal 28:1215-1220. https:// doi.org/10.1016/S0731-7085(02)00021-3

10. Likussar W, Boltz DF (1971) Theory of continuous variations plots and a new method for spectrophotometric determination of extraction and formation constants. Anal Chem 43:1265-1272. https://doi.org/10.1021/ ac60304a006

11. Yoe JH, Jones AL (1944) Colorimetric determination of iron with disodium-1,2-dihydroxybenzene-3,5-disulfonate. Ind Eng Chem Anal Ed 16:111-115. https://doi.org/10.1021/i560126a015

12. Harvey AE, Manning DL (1950) Spectrophotometric methods of establishing empirical formulas of colored complexes in solution. J Am Chem Soc 72:4488-4493. https://doi.org/10.1021/ja01166a044

13. Diehl $H$, Lindstrom $F$ (1959) Eriochrome black $t$ and its calcium and magnesium derivatives. Anal Chem 31:414-418. https://doi.org/10.1021/ ac60147a026
14. Chriswell CD, Schilt AA (1975) New and improved techniques for applying the mole ratio method to the identification of weak complexes in solution. Anal Chem 47:1623-1629

15. Nan Z, Chun-Xiang H (1993) Improved mole ratio method by dual-wavelength spectrophotometry. Analyst 118:1077. https://doi.org/10.1039/ an9931801077

16. Mansour F, Danielson N (2012) Ligand exchange spectrophotometric method for the determination of mole ratio in metal complexes. Microchem J 103:74-78

17. Mabrouk M, Hammad SF, Abdelaziz MA, Mansour FR (2018) Determination of etidronate in pharmaceutical formulations by RP-HPLC method with indirect UV detection. Arab J Med Sci 1:1-4. https://doi.org/10.5455/ ajms.7

18. Mansour FR, Shafi MA, Danielson ND (2012) Flow injection determination of carboxylate, phosphate, and sulfhydryl compounds using metal exchange complexation. Talanta 95:12-17. https://doi.org/10.1016/j.talan ta.2012.03.017

Ready to submit your research? Choose BMC and benefit from

- fast, convenient online submission

- thorough peer review by experienced researchers in your field

- rapid publication on acceptance

- support for research data, including large and complex data types

- gold Open Access which fosters wider collaboration and increased citations

- maximum visibility for your research: over $100 \mathrm{M}$ website views per year

At BMC, research is always in progress.

Learn more biomedcentral.com/submissions 